costs. Repeating the subureteral injections of dextranomer-hyaluronic acid copolymer did not improve the cost-effectiveness of this treatment, except in rare clinical situations.

Benoit and colleagues used costs to the health system and professional fees based on US Medicare rates to make their findings as broadly applicable as possible.

Original article Benoit RM et al. (2006) The costeffectiveness of dextranomer/hyaluronic acid copolymer for the management of vesicoureteral reflux. 2. Reflux correction at the time of diagnosis as a substitute for traditional management. J Urol 176: 2649-2653

\section{Thiazide diuretics arrest the growth of renal calculus fragments}

Nephrolithiasis can persist after treatment with extracorporeal shock-wave lithotripsy (ESWL). Thiazide diuretics reduce the renal excretion of calcium and have been used to treat calcium nephrolithiasis with hypercalciuria; however, concerns remain over the long-term efficacy and adverse effects of thiazide diuretics in this setting. Arrabal-Martín et al. analyzed the evolution of residual nephrolithiasis after ESWL, and evaluated the long-term use of thiazide diuretics in the treatment of nephrolithiasis.

This longitudinal study included 100 patients who presented with residual nephrolithiasis 3 months after ESWL. Patients were randomly allocated to receive either placebo $(n=50)$ or $50 \mathrm{mg}$ hydrochlorothiazide $(n=50)$ daily for 36 months, and were evaluated every 6 months by radiography of the urinary tract, and annually by urinary metabolic studies. Renal ultrasonography was carried out at baseline and study end.

The total expulsion of calculus fragments in the thiazide-treated group was significantly greater than in the placebo-treated group $(P<0.001)$. Markedly more placebo-treated than thiazide-treated patients required at least one additional ESWL procedure (42\% versus $18 \%)$. The incidence of hypercalciuria was similar in both groups, but expulsion of calculus fragments was greater in the thiazide-treated group than in the placebo-treated group $(P=0.001)$. Thiazide diuretic treatment reduced or maintained the size of residual fragments in patients with hypercalciuria; this effect was not observed in placebo-treated patients.
The authors conclude that thiazide administration arrested nephrolithiasis, which favored the spontaneous elimination of residual calculus fragments and reduced the need for additional ESWL procedures.

Original article Arrabal-Martín M et al. (2006) Extracorporeal renal lithotripsy: evolution of residual lithiasis treated with thiazides. Urology 68: 956-959

\section{The lowest PSA value in the year after radiotherapy predicts patient outcome}

The nadir serum PSA level after radiotherapy is a good predictor of patient outcome; however, this value might not occur for several years. Alcántara and colleagues investigated whether the lowest PSA level in the 12 months that followed completion of radiotherapy (PSA levels fall $>90 \%$ within this time) could predict patient outcome.

The authors enrolled 1,000 men with T1-3N0xM0 prostate cancer, who had completed three-dimensional conformal radiotherapy (median dose 76 Gy) a minimum of 24 months before enrollment. Patients were then followed up every 6 months for 5 years, and every 6-12 months thereafter.

The lowest PSA level in the 12 months after radiotherapy independently predicted distant metastasis, overall mortality, prostate-cancerspecific mortality, and biochemical failure in the entire cohort. When a threshold 12-month PSA nadir value of $2 \mathrm{ng} / \mathrm{ml}$ was used, there were marked differences in the 10-year incidences of distant metastasis (19\% versus $4 \%$ ), biochemical failure (46\% versus $30 \%)$, and prostate-cancer-specific mortality $(7 \%$ versus $2 \%)$ for men with values above and below this threshold, respectively.

The authors note that the lowest PSA value in the 12 months following radiotherapy might be more clinically useful than PSA doubling time as a predictor of outcome, because PSA doubling time can only be calculated for a subset of patients. They conclude that the 12-month PSA nadir after radiotherapy could potentially identify patients at high risk of progression to biochemical failure, distant metastasis, and mortality, and has potential for use as a surrogate end point in clinical trials.

Original article Alcántara P et al. (2006) Prostratespecific antigen nadir within 12 months of prostrate cancer radiotherapy predicts metastasis and death. Cancer 28: 41-47 